\title{
Stabilization of a Class of Switched Positive Nonlinear Systems
}

\author{
Xing Xing, ${ }^{1,2}$ Zhichun Jia, ${ }^{3}$ Yunfei Yin, ${ }^{1}$ and Tingting $\mathrm{Wu}^{1}$ \\ ${ }^{1}$ College of Engineering, Bohai University, Jinzhou, Liaoning 121013, China \\ ${ }^{2}$ Space Control and Inertial Technology Research Center, Harbin Institute of Technology, Harbin, Heilongjiang 150001, China \\ ${ }^{3}$ College of Information Science and Technology, Bohai University, Jinzhou, Liaoning 121013, China
}

Correspondence should be addressed to Xing Xing; superfather.x@gmail.com

Received 22 September 2014; Accepted 5 December 2014

Academic Editor: Guangming Xie

Copyright (C) 2015 Xing Xing et al. This is an open access article distributed under the Creative Commons Attribution License, which permits unrestricted use, distribution, and reproduction in any medium, provided the original work is properly cited.

\begin{abstract}
The problem of switching stabilization for a class of switched positive nonlinear systems (switched positive homogeneous cooperative system (SPHCS) in the continuous-time context and switched positive homogeneous order-preserving system (SPHOS) in the discrete-time context) is studied by using average dwell time (ADT) approach, where the positive subsystems are possibly all unstable. To tackle this problem, a new class of ADT switching is first defined, which is different from the previous defined ADT switching in the literature. Then, the proposed ADT is designed via analyzing the weighted $l_{\infty}$ norm of the considered system's state. A sufficient condition of stabilization for SPHCSs with unstable positive subsystems is derived in continuous-time context. Furthermore, a sufficient condition for SPHOSs under the assumption that all modes are possibly unstable is also obtained. Finally, a numerical example is given to demonstrate the advantages and effectiveness of our developed results.
\end{abstract}

\section{Introduction}

Switched system is a class of hybrid systems that involves a coupling between continuous dynamics and discrete events, which has wide application areas such as traffic control, process control, network control systems, automotive industry, and mechanical systems. Due to their numerous applications, switched systems have been a hot research topic in the past decades [1-5]. Most efforts in researches of switched systems are mainly devoted to the dynamic behavior analysis and property characterization, for example, controllability, observability, realizability, optimized performance, and especially the stability. The authors in [6] applied a class of Lyapunov-like function to study the problem of stability for switched systems comprising unstable subsystems.

On the other hand, many physical systems encountered in practice involve state variables that are always confined to be nonnegative. For instance, density of a object, absolute temperatures, and concentration of substances in chemical processes are always positive. Such systems are generally termed as positive systems whose states and output are positive whenever the initial conditions and input are nonnegative [7]. Numerous models having positive behavior can be found in many areas such as biology, sociology, and communication networks. Given their practical importance, recently, positive system has been paid much attention by a large number of researchers [8-14]. As it is well known, positivity of the system state can yield some interesting properties and bring about some efficiently tackling techniques to positive linear systems $[7,15]$. For example, the so-called copositive linear Lyapunov function with positive vector parameters can be particularly chosen as a system energy function for switched positive linear systems. In the most recent few years, positive nonlinear systems have also been noticed due to their important applications [16-18]. A valuable issue naturally arises: whether those properties, results, and methods for positive linear systems can be extended to positive nonlinear systems. In [19], the authors proved that, same to positive linear systems with constant delay, the stability of homogeneous cooperative system is also independent of delays. Afterwards, the authors of [20] extended this fact to continuous-time homogeneous cooperative systems and discrete-time homogeneous monotone systems with bounded time-varying delays.

Furthermore, switched positive system that possesses the essences of both switched systems and positive systems is 
recently studied due to its wide applications in practice. As a special class of switched systems and positive systems, some relevant methods applicable to positive systems or switched systems are still suitable for switched positive systems [21,22]. But anyway, it should be pointed out that those methods may not be as efficient as they are for general switched systems or positive systems. Therefore, many researchers have recently focused on exploring efficient approaches and results for switched positive systems. The authors of [23] introduced a multiple linear copositive Lyapunov function (MLCLF) to investigate the problem of stability for switched positive systems. Then, the authors of [24] presented a generalization of copositive types of Lyapunov function for stability analysis of switched positive systems. Note that those works all consider switched positive linear systems. So far there are few results reported for switched positive nonlinear systems which are however of both theoretical and practical importances.

As far as the stabilization of switched positive systems is concerned, how to find an appropriate switching signals to guarantee system stability is an important issue. Switching signals that may be either autonomous or controlled generally include arbitrary switching signals, stochastic switching signals, and constraint switching signals. Time constrained switching signal that is viewed as an important class of constraint switching signals naturally has been extensively investigated during the past several decades because of its strong applicability. Time constrained switching signal can be also called slow switching, and it is classified into three types: dwell time (DT), average dwell time (ADT), and modedependent average dwell time switching (MDADT).

In the recent few years, time constrained switching has been successfully used for switching stabilization of both general switched systems and switched positive systems. To list a few, when all the subsystems of a switched linear system are stable, the author of [25] had shown that the system is exponentially stable if the DT is sufficiently large. It was proved in [26-28] that ADT turns out to be very useful for analysis and synthesis of switched systems. The ADT was extended to MDADT in [29] for stabilization of general switched systems. In [23], the ADT switching was utilized for switched positive linear systems. It is noted that, most recently, the time constrained switching stabilization for switched (positive) linear systems with partly/all unstable subsystems have been paid some attentions [6, 30, 31]. However, such an attempt is just a start, and the slow switching stabilization for switched (positive) systems with possibly all unstable subsystems has not been fully solved, especially for switched positive nonlinear systems.

In summary, the existing time constrained switching stabilization results for switched positive systems are mainly focused on the linear case, and those promising ideas therein are not applicable for switched positive nonlinear systems. Such a problem becomes more complicated once all the nonlinear subsystems become unstable. To the best of the authors' knowledge, up to now, there is no literature reporting the switching stabilization problem for switched positive nonlinear systems with possibly all unstable subsystems, even in the linear case.
All the above observations motivate us to carry out the present work. Compared with some existing results, our results can solve the switching stabilization problem of switched positive nonlinear systems though all the subsystems are unstable. The most important of the paper lies in that a new class of ADT is proposed and the weighted $l_{\infty}$ norm of the considered system's state is introduced to tackle the considered problem. The layout of the paper is organized as follows. Section 2 reviews some necessary definitions of switched positive nonlinear systems and defines a new concept of ADT which characterizes a different set of switching signals from traditional ADT. In Section 3, stabilization criteria for switched positive nonlinear systems with possibly all unstable subsystems via proposed ADT switching are derived in continuous-time and discrete-time case. Section 4 provides a numerical example to demonstrate the feasibility and effectiveness of the proposed techniques, and Section 5 concludes the paper.

Notations. In this paper, the notations used are standard. $\mathbb{R}$, $\mathbb{R}^{n}$, and $\mathbb{R}_{+}^{n}$ denote the field of real numbers, $n$-dimensional Euclidean space, and the nonnegative orthant of $\mathbb{R}^{n}$, respectively, and $\mathbb{R}_{0}^{n}$ stands for $\mathbb{R}_{+}^{n} \backslash\left\{0^{n}\right\}$, and Metzler is a matrix whose off diagonal entries are nonnegative. The notation $\|\cdot\|$ refers to the Euclidean norm. For $\mathbf{x} \in \mathbb{R}^{n}, x_{i}$ denotes the $i$ th component of $\mathbf{x}$. In addition, $\mathbf{x} \geq \mathbf{y}$ (or $\mathbf{x}>\mathbf{y}$ ) mean that all entries of vector $x_{i} \geq y_{i}$ (or $x_{i}>y_{i}$ ).

\section{Problem Formulation and Preliminaries}

This section presents some definitions and preliminary results which will be used throughout the paper. We first recall some preliminaries about positive nonlinear systems for the introduction of switched positive nonlinear systems hereafter. Consider the following nonlinear system:

$$
\vartheta \mathbf{x}(t)=\mathbf{f}(x(t)),
$$

where $\mathbf{x}(t) \in \mathbb{R}^{n}$ is the state vector; $\mathbf{f}: \mathbb{R}^{n} \rightarrow \mathbb{R}^{n}$ is a smooth functions vector, and is homogeneous cooperative in the continuous-time case (homogeneous order-preserving in the discrete-time case); the symbol $\vartheta$ denotes the derivative operator in the continuous-time context $\vartheta \mathbf{x}(t)=(d / d t) \mathbf{x}(t)$ and the shift forward operator in the discrete-time case $\vartheta \mathbf{x}(t)=\mathbf{x}(t+1)$.

Definition 1. System (1) is said to be positive if and only if, for every nonnegative initial state, its state is nonnegative.

Definition 2. A continuous vector field $\mathbf{f}: \mathbb{R}^{n} \rightarrow \mathbb{R}^{n}$ is said to be homogeneous if, for all $\mathbf{x}(t) \in \mathbb{R}^{n}$, and all real $\lambda>0$, $\mathbf{f}(\lambda \mathbf{x})=\lambda \mathbf{f}(\mathbf{x})$.

Definition 3. A continuous vector field $\mathbf{f}: \mathbb{R}^{n} \rightarrow \mathbb{R}^{n}$, which is $\mathscr{C}^{1}$ on $\mathbb{R}^{n} \backslash\{\mathbf{0}\}$, is said to be cooperative if the Jacobian $(\partial \mathbf{f} / \partial \mathbf{x})(\mathbf{a})$ is Metzler for all $\mathbf{a} \in \mathbb{R}_{0}^{n}$.

Definition 4. A continuous vector field $\mathbf{f}: \mathbb{R}^{n} \rightarrow \mathbb{R}^{n}$ is said to be order-preserving on $\mathbb{R}_{+}^{n}$, if $\mathbf{f}(\mathbf{x})>\mathbf{f}(\mathbf{y})$ for any $\mathbf{x}, \mathbf{y} \in \mathbb{R}_{+}^{n}$ such that $\mathbf{x} \geq \mathbf{y}$. 
Lemma 5. If $\mathbf{f}: \mathbb{R}^{n} \rightarrow \mathbb{R}^{n}$ is cooperative, then for any two vectors $\mathbf{x}, \mathbf{y} \in \mathbb{R}_{0}^{n}$ satisfying $\mathbf{x} \geq \mathbf{y}$ and $x_{i}=y_{i}, f_{i}(\mathbf{x}) \geq f_{i}(\mathbf{y})$.

Remark 6. It should be pointed out that, if $\mathbf{f}$ is defined by homogeneous and cooperative vector field in the continuoustime context (or $\mathbf{f}$ is defined by homogeneous and orderpreserving vector field in the discrete-time case), the nonlinear system (1) is positive, which means that for every nonnegative initial condition $\mathbf{x}(0) \in \mathbb{R}_{+}^{n}$, the corresponding state trajectory $\mathbf{x}(t) \in \mathbb{R}_{+}^{n}$ for all $t \geq 0$.

In this paper, we consider the following SPHCS (SPHOS in the discrete-time case) consisting of a family of subsystems (1):

$$
\vartheta \mathbf{x}(t)=\mathbf{f}_{\sigma(t)}(\mathbf{x}(t)),
$$

where $\mathbf{x}(t) \in \mathbb{R}^{n}$ is the state vector; $\sigma(t)$ is a switching signal which is a piecewise constant function from the right of time and takes its values in the finite set $S=\{1, \ldots, m\}$, where $m>1$ is the number of subsystems. $\mathbf{f}_{p}: \mathbb{R}^{n} \rightarrow \mathbb{R}^{n}$ are smooth functions for any $\sigma(t)=p \in S$. Moreover, all the subsystems in system (1) may be unstable. Also, for a switching sequence, $0<t_{1}<\cdots<t_{p}<t_{p+1}<\cdots, \sigma(t)$ may be either autonomous or controlled. When $t \in\left[t_{p}, t_{p+1}\right)$, we say $\sigma\left(t_{p}\right)$ th mode is active. With respect to switching law $\sigma(t)$, the following exponential stability definition of system (2) is given, and we denote time by $k$ in the discrete-time case.

Definition 7. Switched system (2) with switching $\sigma(t)$ is said to be globally uniformly exponentially stable (GUES), if there exist constants $\alpha>0, \beta>0$ (resp., $0<\lambda<1$ ), such that the solution of the system satisfies $\|\mathbf{x}(t)\| \leq \alpha e^{-\beta\left(t-t_{0}\right)}\left\|\mathbf{x}\left(t_{0}\right)\right\|$, $\forall t \geq t_{0}$ (resp., $\|\mathbf{x}(k)\| \leq \alpha \lambda^{-\left(k-k_{0}\right)}\left\|\mathbf{x}\left(t_{0}\right)\right\|, \forall k \geq k_{0}$, with any initial conditions $\mathbf{x}\left(t_{0}\right)$ ( or $\left.\mathbf{x}\left(k_{0}\right)\right)$ ).

Due to the fact that all the positive nonlinear subsystems of system (2) may be unstable, we need to design time constrained switching sequences, such that the switched nonlinear system (2) is GUES. In order to achieve this goal, let us first define the following ADT switching.

Definition 8. For a switching signal $\sigma(t)$ and each $t_{2} \geq t_{1} \geq 0$, let $N_{\sigma}\left(t_{2}, t_{1}\right)$ denote the number of discontinuities of $\sigma(t)$ in the interval $\left(t_{1}, t_{2}\right)$. One says that $\sigma(t)$ has an average dwell time $\tau_{a}$ if there exist two positive numbers $N_{0}$ and $\tau_{a}$ such that

$$
N_{\sigma}\left(t_{2}, t_{1}\right) \geq N_{0}+\frac{t_{2}-t_{1}}{\tau_{a}}, \quad \forall t_{2} \geq t_{1} \geq 0 .
$$

\section{Main Results}

In this section, we consider the problem of stabilization for switched positive nonlinear system (2) with our proposed ADT switching.

First, we are in a position to provide the continuous-time version of stabilization condition in the case that the $\mathbf{f}_{p}$ is defined in homogeneous and cooperative vector field.
Theorem 9 (continuous-time version). Consider SPHCS (2) possibly composed of all unstable modes. If there exist a set of vectors $\nu_{p}>0, p \in S$ and two positive numbers $\eta>0$ and $0<\mu<1$ such that $\forall i=1,2, \ldots, n, \forall p \in S$

$$
\begin{gathered}
\mathbf{f}_{p}\left(v_{p}\right)>0, \\
\frac{f_{p i}\left(v_{p i}\right)}{v_{p i}}-\eta<0, \\
\left\|\mathbf{x}\left(t_{p}^{+}\right)\right\|_{\mathbf{v}_{p}}^{\infty}-\mu\left\|\mathbf{x}\left(t_{p}^{-}\right)\right\|_{\mathbf{v}_{q}}^{\infty} \leq 0,
\end{gathered}
$$

where the $\left\|x\left(t_{p}\right)\right\|_{v_{p}}^{\infty}$ is the weight $l_{\infty}$ norm defined as

$$
\left\|\mathbf{x}\left(t_{p}\right)\right\|_{v_{p}}^{\infty}=\max _{1 \leq i \leq n} \frac{\left|x\left(t_{p}\right)_{i}\right|}{v_{i}},
$$

then SPHCS (2) is globally uniformly exponentially stable for every switching signal $\sigma(t)$ with average dwell time:

$$
\tau_{a} \leq \tau_{a}^{*}=\frac{-\ln \mu}{\eta} .
$$

Proof. For any $T>0, t_{0}=0$, we denote $t_{1}, t_{2}, \ldots, t_{p}$, $t_{p+1}, \ldots, t_{N_{N_{\sigma(T, 0)}}}$ as the switching times on time interval $[0, T]$. Then, we consider the function

$$
W(t)=e^{-\eta\left(t-t_{\sigma(t)}\right)}\|\mathbf{x}(t)\|_{\boldsymbol{v}_{\sigma(t)}}^{\infty} .
$$

It is immediately clear that $W(t)$ is a continuously differentiable function. When $t \in\left[t_{p}, t_{p+1}\right)$, we get from (9) that

$$
W(t)=e^{-\eta\left(t-t_{p}\right)}\|\mathbf{x}(t)\|_{\nu_{p}}^{\infty} .
$$

It will show that $W(t)$ is nonincreasing when $t \in\left[t_{p}, t_{p+1}\right)$. Next, we suppose that there exists a point-in-time $\widehat{t} \epsilon$ $\left[t_{p}, t_{p+1}\right]$, such that

$$
W(\widehat{t})=\left\|\mathbf{x}\left(t_{p}\right)\right\|_{\nu_{p}}^{\infty}
$$

Then, one can obtain from (7) and (11) that

$$
W(\hat{t})=e^{-\eta\left(t-t_{\sigma}(t)\right)} \max _{1 \leq i \leq n} \frac{\left|x_{i}(\hat{t})\right|}{v_{p i}}=e^{-\eta\left(t-t_{p}\right)} \frac{x_{k}(\hat{t})}{v_{p k}} .
$$

This together with (11) yields

$$
\begin{aligned}
x_{k}(\hat{t}) & =\|\mathbf{x}(\hat{t})\|_{v_{p}}^{\infty} v_{p k} \\
& =e^{\eta\left(\hat{t}-t_{p}\right)}\left\|\mathbf{x}\left(t_{p}\right)\right\|_{v_{p}}^{\infty} v_{p k}, \\
\mathbf{x}(\hat{t}) & \preceq\|\mathbf{x}(\hat{t})\|_{v_{p}}^{\infty} v_{p} \\
& =e^{\eta\left(\hat{t}-t_{p}\right)}\left\|\mathbf{x}\left(t_{p}\right)\right\|_{v_{p}}^{\infty} v_{p} .
\end{aligned}
$$


Moreover, since $\mathbf{f}_{p}: \mathbb{R}^{n} \rightarrow \mathbb{R}^{n}$ and $p \in S$ is in homogeneous and cooperative vector field, based on Lemma 5 , it can be derived from (5), (12), and (13) that

$$
\begin{aligned}
\dot{W}(t)= & e^{-\eta\left(\hat{t}-t_{p}\right)} \frac{f_{p k}(\mathbf{x}(\hat{t}))}{v_{p k}}-\eta e^{-\eta\left(\hat{t}-t_{p}\right)} \frac{x_{k}(\widehat{t})}{v_{p k}} \\
\leq & e^{-\eta\left(\hat{t}-t_{p}\right)} \frac{f_{p k}\left(e^{\eta\left(\hat{t}-t_{p}\right)}\left\|\mathbf{x}\left(t_{p}\right)\right\|_{\nu_{p}}^{\infty} v_{p}\right)}{v_{p k}} \\
& -\eta e^{-\eta\left(\hat{t}-t_{p}\right)} \frac{e^{\eta\left(\hat{t}-t_{p}\right)}\left\|\mathbf{x}\left(t_{p}\right)\right\|_{\nu_{p}}^{\infty} v_{p k}}{v_{p k}} \\
= & e^{-\eta\left(\hat{t}-t_{p}\right)} \frac{e^{\eta\left(\hat{t}-t_{p}\right)}\left\|\mathbf{x}\left(t_{p}\right)\right\|_{\nu_{p}}^{\infty} f_{p k}\left(v_{p}\right)}{v_{p k}}-\eta\|\mathbf{x}(\widehat{t})\|_{v_{p}}^{\infty} \\
= & \left\|\mathbf{x}\left(t_{p}\right)\right\|_{\nu_{p}}^{\infty}\left(\frac{f_{p k}\left(v_{p}\right)}{v_{p k}}-\eta\right)<0 .
\end{aligned}
$$

Note that if (11) holds, we can get $\dot{W}(\hat{t})<0$, which implies that the upper-right Dini derivative $D^{+}(W(\hat{t}))<0$. Thus, it can be seen that $W(t)$ is nonincreasing when $t \in\left[t_{p}, t_{p+1}\right)$. This together with (10) gives that for $\forall t \in\left[t_{p}, t_{p+1}\right)$

$$
\begin{aligned}
W(t) & =e^{-\eta\left(t-t_{p}\right)}\|\mathbf{x}(t)\|_{\nu_{p}}^{\infty} \\
& \leq W\left(t_{p}\right) \\
& =\left\|\mathbf{x}\left(t_{p}\right)\right\|_{\nu_{p}}^{\infty} .
\end{aligned}
$$

According to (6) and (15), one can obtain that

$$
\begin{aligned}
\left\|\mathbf{x}\left(t_{p+1}^{+}\right)\right\|_{v_{p}}^{\infty} & \leq \mu\left\|\mathbf{x}\left(t_{p+1}^{-}\right)\right\|_{\nu_{p}}^{\infty} \\
& \leq \mu e^{\eta\left(t_{p+1}-t_{p}\right)}\left\|\mathbf{x}\left(t_{p}\right)\right\|_{v_{p}}^{\infty} .
\end{aligned}
$$

By integrating this for $t \in\left[t_{p}, t_{p+1}\right)$, one gets that

$$
\begin{aligned}
\left\|\mathbf{x}\left(T^{-}\right)\right\|_{\nu_{N_{\sigma}}}^{\infty} & \leq e^{\eta\left(T-t_{N_{\sigma}}\right)}\left\|\mathbf{x}\left(t_{N_{\sigma}}\right)\right\|_{\nu_{N_{\sigma}}}^{\infty} \\
& =e^{\eta\left(T-t_{N_{\sigma}}\right)}\left\|\mathbf{x}\left(t_{N_{\sigma}}^{+}\right)\right\|_{\nu_{N_{\sigma}}}^{\infty} \\
& \leq \mu e^{\eta\left(T-t_{N_{\sigma}-1}\right)}\left\|\mathbf{x}\left(t_{N_{\sigma}}\right)\right\|_{\nu_{N_{\sigma}-1}}^{\infty} \\
\vdots & \\
& \leq \mu^{N_{\delta}} e^{\eta T}\|\mathbf{x}(0)\|_{\nu_{0}}^{\infty} .
\end{aligned}
$$

Moreover, it can be derived from (3) and (17) that

$$
\begin{aligned}
\|\mathbf{x}(T)\|_{\nu_{N_{\sigma}}}^{\infty} & \leq \mu^{N_{\delta}} e^{\eta T}\|\mathbf{x}(0)\|_{\nu_{0}}^{\infty} \\
& \leq e^{\eta T} e^{\left(N_{0}+T / \tau_{a}\right) \ln \mu}\|\mathbf{x}(0)\|_{\nu_{0}}^{\infty} \\
& =e^{N_{0} \ln \mu} e^{\left(\eta+\ln \mu / \tau_{a}\right) T}\|\mathbf{x}(0)\|_{\nu_{0}}^{\infty} .
\end{aligned}
$$

Therefore, by denoting $\alpha=e^{N_{0} \ln \mu}, \beta=-\left(\eta+\mu / \tau_{a}\right)$, one can get from (18) that

$$
\|x(T)\| \leq \alpha e^{-\beta T}\|x(0)\|, \quad \forall T \geq 0 .
$$

By Definition 7, we conclude that SPHCS (2) is GUES by our proposed ADT switching signals (3) satisfying (8) if the conditions (4)-(6) hold. This completes the proof.

Remark 10. It has been shown in [19] that if there exists a vector $\boldsymbol{v}_{p}>0$ satisfying $\mathbf{f}(\boldsymbol{v})>0$, the corresponding homogenous cooperative system is not globally asymptotically stable. Thus, all the homogenous cooperative subsystems of SPHCS (2) may be unstable, if (4) holds. Under the ADT designed in Theorem 9, the stabilization can be achieved even if all the subsystems are unstable. On the other hand, it should be pointed out that, different from the existing results where the lower bound of the ADT should be specified a priori, we need to set the upper bound in advance in our results.

Next, the following theorem is provided for the discretetime version of stabilization condition for SPHOS (2) where the $\mathbf{f}_{p}$ is defined in homogeneous and order-preserving vector field.

Theorem 11 (discrete-time version). Consider SPHOS (2) possibly composed of all unstable modes. If there exist a set of vectors $\boldsymbol{\nu}_{p}>0, p \in S$ and two positive numbers $0<\eta<1$ and $0<\mu<1$ such that $\forall i=1,2, \ldots, n, \forall p \in S$

$$
\begin{gathered}
\mathbf{f}_{p}\left(\boldsymbol{v}_{p}\right)>\boldsymbol{v}_{p}, \\
\frac{f_{p i}\left(v_{p i}\right)}{v_{p i}}-\frac{1}{\eta} \leq 0, \\
\left\|\mathbf{x}\left(k_{p}^{+}\right)\right\|_{\mathbf{v}_{p}}^{\infty}-\mu\left\|\mathbf{x}\left(k_{p}^{-}\right)\right\|_{\mathbf{v}_{q}}^{\infty} \leq 0,
\end{gathered}
$$

where the $\left\|x\left(t_{p}\right)\right\|_{v_{p}}^{\infty}$ is the weight $l_{\infty}$ norm defined as

$$
\left\|\mathbf{x}\left(t_{p}\right)\right\|_{v_{p}}^{\infty}=\max _{1 \leq i \leq n} \frac{\left|x\left(t_{p}\right)_{i}\right|}{v_{i}},
$$

then SPHOS (2) is globally uniformly exponentially stable for every switching signal $\sigma$ with average dwell time:

$$
\tau_{a} \leq \tau_{a}^{*}=\frac{\ln \mu}{\ln \eta}
$$

Proof. For any $K>0, k_{0}=0$, we denote $k_{1}, k_{2}, \ldots, k_{p}$, $k_{p+1}, \ldots, k_{N_{N_{\sigma(K, 0)}}}$ as the switching times on time interval $[0, T]$. Then, we consider the function

$$
W(t)=\eta^{k-k_{\sigma(k)}}\|\mathbf{x}(k)\|_{v_{\sigma(k)}}^{\infty} .
$$

When $k \in\left[k_{p}, k_{p+1}\right)$, we get from (25) that

$$
W(k)=\eta^{k-k_{p}}\|\mathbf{x}(k)\|_{v_{p}}^{\infty} .
$$


Next, it will be shown that $W(k) \leq\left\|\mathbf{x}\left(k_{p}\right)\right\|_{\nu p}^{\infty}$ when $k \in$ $\left[k_{p}, k_{p+1}\right)$. First, when $k=k_{p}$, the above inequality obviously holds. Then, we suppose that $k>k_{p}$; for a given $k \in\left[k_{p}, k_{p+1}\right)$ the above inequality is satisfied and it proves that it is also correct for $k+1 \in\left(k_{p}, k_{p+1}\right)$. Due to the fact that for $k \in$ $\left[k_{p}, k_{p+1}\right), W(k) \leq\left\|\mathbf{x}\left(k_{p}\right)\right\|_{p p}^{\infty}$ is true, one can obtain that

$$
W(k)=\eta^{k-k_{p}} \max _{1 \leq i \leq n} \frac{\left|x_{i}(k)\right|}{v_{p i}} \leq\left\|\mathbf{x}\left(k_{p}\right)\right\|_{v_{p}}^{\infty},
$$

which implies that

$$
\mathbf{x}(k) \preceq \eta^{k_{p}-k}\left\|\mathbf{x}\left(k_{p}\right)\right\|_{v_{p}}^{\infty} v_{p} .
$$

Moreover, since $\mathbf{f}_{p}: \mathbb{R}^{n} \rightarrow \mathbb{R}^{n}$ and $p \in S$ is defined in homogeneous and order-preserving vector field, it can be seen from (21), (26), and (28) that

$$
\begin{aligned}
W(k+1) & =\eta^{k+1-k_{p}}\left\|\mathbf{x}\left((k+1)_{p}\right)\right\|_{\nu_{p}}^{\infty} \\
& =\eta^{k+1-k_{p}}\left\|\mathbf{f}_{\mathbf{p}}(\mathbf{x}(k))\right\|_{\nu_{p}}^{\infty} \\
& \leq \eta^{k+1-k_{p}}\left\|\mathbf{f}_{\mathbf{p}}\left(\eta^{k_{p}-k}\left\|\mathbf{x}\left(k_{p}\right)\right\|_{v_{p}}^{\infty} v_{p}\right)\right\|_{\nu_{p}}^{\infty} \\
& =\eta^{k+1-k_{p}} \eta^{k_{p}-k}\left\|\mathbf{x}\left(k_{p}\right)\right\|_{\nu_{p}}^{\infty}\left\|\mathbf{f}_{\mathbf{p}}\left(v_{p}\right)\right\|_{\nu_{p}}^{\infty} \\
& =\eta\left\|\mathbf{x}\left(k_{p}\right)\right\|_{\nu_{p}}^{\infty}\left\|\mathbf{f}_{\mathbf{p}}\left(v_{p}\right)\right\|_{\nu_{p}}^{\infty} \\
& =\eta \max _{1 \leq i \leq n} \frac{\left|f_{p i}\left(v_{p}\right)\right|}{v_{p i}}\left\|\mathbf{x}\left(k_{p}\right)\right\|_{v_{p}}^{\infty} \\
& \leq\left\|\mathbf{x}\left(k_{p}\right)\right\|_{v_{p}}^{\infty} .
\end{aligned}
$$

Thus, we can obtain that (27) is right for $\forall k \in\left[t_{p}, t_{p+1}\right)$. Combining this together with (22), one can get

$$
\begin{aligned}
\left\|\mathbf{x}\left(k_{p+1}^{+}\right)\right\|_{\nu_{p}}^{\infty} & \leq \mu\left\|\mathbf{x}\left(k_{p+1}^{-}\right)\right\|_{v_{p}}^{\infty} \\
& \leq \mu \eta^{k_{p+1}-k_{p}}\left\|\mathbf{x}\left(k_{p}\right)\right\|_{\nu_{p}}^{\infty} .
\end{aligned}
$$

Similar to (17) and (18), we have for $\forall K>0$

$$
\begin{aligned}
\left\|\mathbf{x}\left(K^{-}\right)\right\|_{\nu_{N_{\sigma}}}^{\infty} & \leq \mu^{N_{\delta}} \eta^{-K}\|\mathbf{x}(0)\|_{\nu_{0}}^{\infty} \\
& \leq \exp \left\{\left(\frac{K}{\tau_{a}}+N_{0}\right) \ln \mu\right\} \exp \{-K \ln \eta\}\|\mathbf{x}(0)\|_{\nu_{0}}^{\infty} \\
& =\exp \left\{N_{0} \ln \mu\right\} \exp \left\{\left(\frac{\ln \mu}{\tau_{a}}-\ln \eta\right) K\right\}\|\mathbf{x}(0)\|_{\nu_{0}}^{\infty} .
\end{aligned}
$$

Therefore, by denoting $\alpha=\exp \left\{N_{0} \ln \mu\right\}, \lambda=$ $\exp \left\{\left(\ln \mu / \tau_{a}-\ln \eta\right)\right\}$; one can get from (31) that

$$
\|x(K)\| \leq \alpha \lambda^{K}\|x(0)\|, \quad \forall K \geq 0 .
$$

We conclude that SPHOS (2) is GUES by our proposed ADT switching signals (3) satisfying (24) if the conditions (20)(22) hold. This completes the proof.

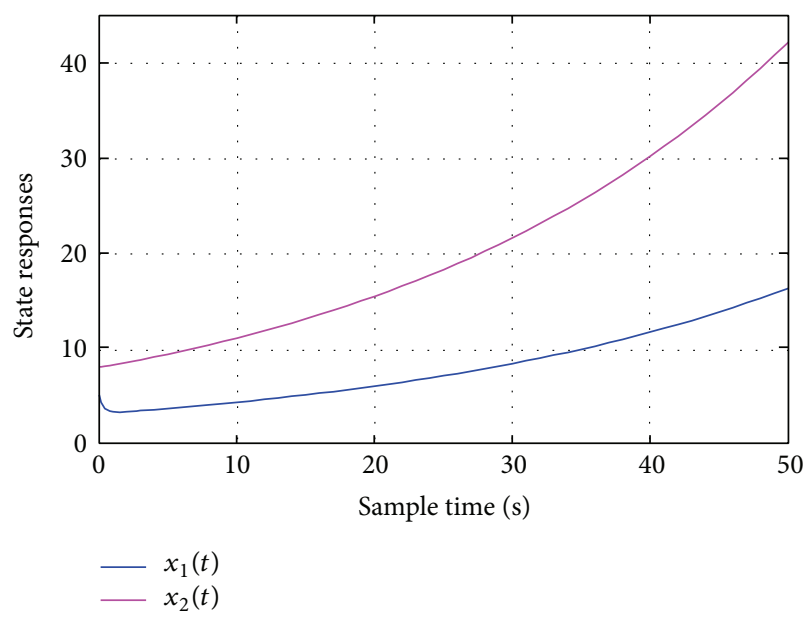

FIGURE 1: State response of the subsystem $\Sigma_{1}$.

Remark 12. In Theorems 9 and 11, based on a novel proposed switching signals, sufficient conditions of stabilization for a class of switched positive nonlinear systems are obtained, where the positive subsystems are possibly all unstable.

\section{A Numerical Example}

We provide the following numerical example in this section to verify our main results developed in this paper.

Example 1. Consider a switched nonlinear system consisting of two homogeneous cooperative subsystems described by

$$
\begin{aligned}
& \Sigma_{1}=\left\{\begin{aligned}
\dot{x}_{1}(t)= & f_{11}\left(x_{1}(t), x_{2}(t)\right) \\
= & -2 x_{1}(t)+4 x_{2}(t)-3 \sqrt{x_{1}^{2}(t)+x_{2}^{2}(t),} \\
\dot{x}_{2}(t)= & f_{12}\left(x_{1}(t), x_{2}(t)\right) \\
= & -0.2 x_{1}(t)+0.1 x_{2}(t) \\
& +0.01 \sqrt{x_{1}^{2}(t)+x_{2}^{2}(t),}
\end{aligned}\right. \\
& \Sigma_{2}=\left\{\begin{aligned}
\dot{x}_{1}(t)= & f_{21}\left(x_{1}(t), x_{2}(t)\right) \\
= & -2 x_{1}(t)+3 x_{2}(t)+1.2 \sqrt{x_{1}^{2}(t)+x_{2}^{2}(t)}, \\
\dot{x}_{2}(t)= & f_{22}\left(x_{1}(t), x_{2}(t)\right) \\
= & 1.2 x_{1}(t)-x_{2}(t)+0.6 \sqrt{x_{1}^{2}(t)+x_{2}^{2}(t)} .
\end{aligned}\right.
\end{aligned}
$$

It is seen in (33) that $\mathbf{f}_{1}, \mathbf{f}_{2}$ are defined in homogeneous cooperative vector field, which means that the switched nonlinear system is a switched positive system. There exist a vector $\boldsymbol{\nu}_{1}=[3,7.8]>0$ with $\mathbf{f}\left(\boldsymbol{\nu}_{1}\right)=[0.1289,0.2636]>0$ and a vector $\boldsymbol{\nu}_{2}=[3,1.2]>0$ with $\mathbf{f}\left(\boldsymbol{v}_{2}\right)=[1.8754,0.4613]>0$. Thus, both of them are unstable, and the state trajectories are shown in Figures 1 and 2.

Next, we are interested in designing a kind of switching signal $\sigma(t)$ with property (3) to asymptotically stabilize the system. Furthermore, we generate two possible switching sequences by $\tau_{a}=0.5$ and $\tau_{a}=2$, respectively; the corresponding state responses of the system under initial state 


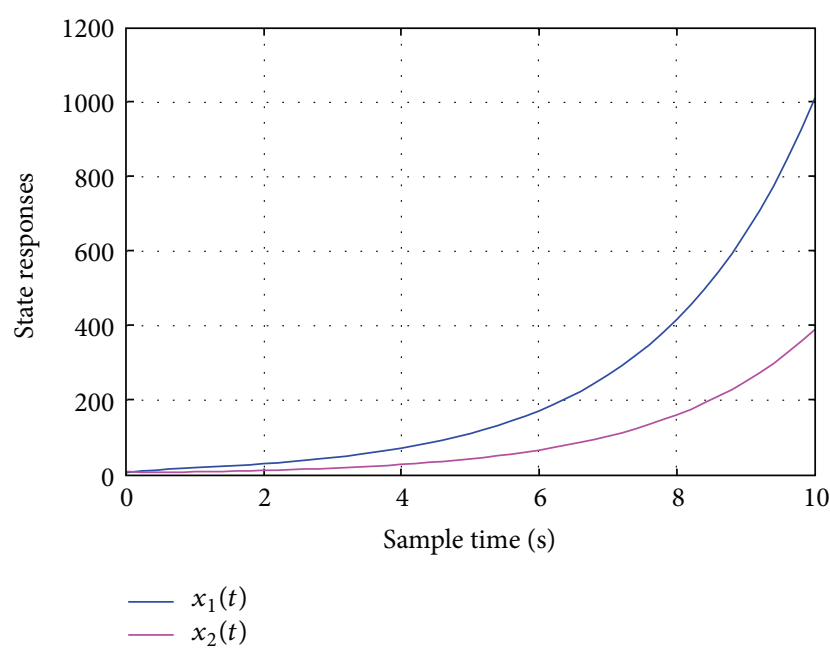

FIGURE 2: State response of the subsystem $\Sigma_{2}$.

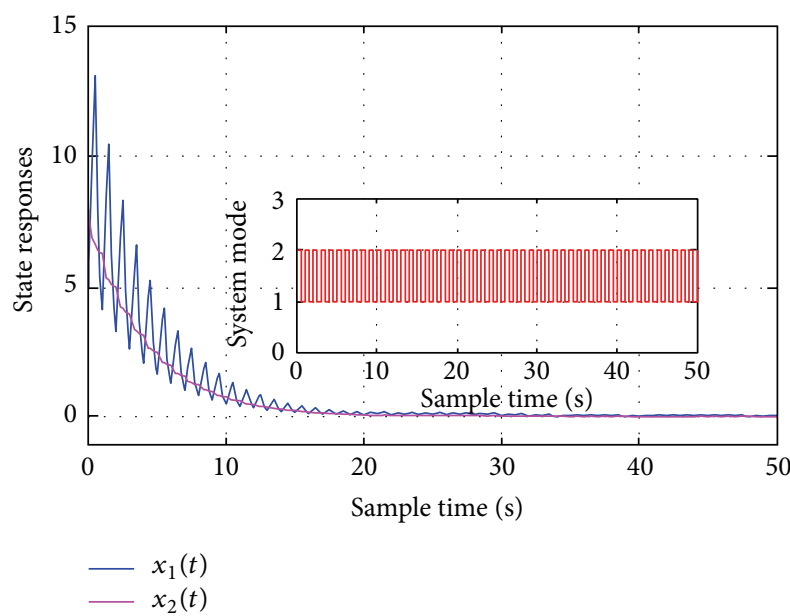

FIGURE 3: State responses of switched nonlinear system (33) under switching signal $\sigma(t)$ with $\tau_{a}=0.5$.

condition $x(0)=\left[\begin{array}{ll}10 & 15\end{array}\right]^{T}$ are shown in Figures 3 and 4, from which we can see that the switched nonlinear system is stable under $\tau_{a}=0.5$ but unstable under $\tau_{a}=2$.

\section{Conclusions}

The stabilization problem for switched positive nonlinear systems composed of possibly all unstable subsystems are studied in both continuous-time and discrete-time domains by using ADT switching. As a first attempt, a new class of ADT switching signal is proposed, and then it is designed for the system via the analysis of the weight $l_{\infty}$ norm of the system. Two sufficient stabilization conditions for the underlying systems are derived. The highlight of the paper lies in that it is the first time the stabilization is solved for our considered switched positive nonlinear system where the system is possibly composed of all unstable subsystems. A numerical example is provided to show the effectiveness of our proposed approach. In our future work, we aim at

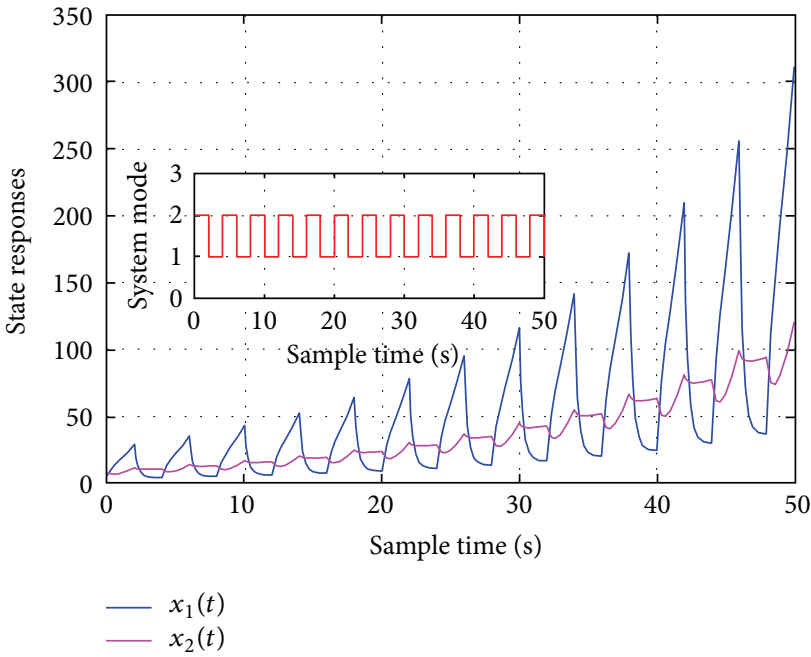

FIGURE 4: State responses of switched nonlinear system (33) under switching signal $\sigma(t)$ with $\tau_{a}=2$.

developing numerially easily checked conditions for the considered systems, and the problem of stabilization for switched positive nonlinear system with delays also needs to be studied.

\section{Conflict of Interests}

The authors declare that there is no conflict of interests regarding the publication of this paper.

\section{References}

[1] Y. Kao, C. Wang, F. Zha, and H. Cao, "Stability in mean of partial variables for stochastic reaction-diffusion systems with Markovian switching," Journal of the Franklin Institute, vol. 351, no. 1, pp. 500-512, 2014.

[2] X.-M. Sun, W. Wang, G.-P. Liu, and J. Zhao, "Stability analysis for linear switched systems with time-varying delay," IEEE Transactions on Systems, Man, and Cybernetics Part B: Cybernetics, vol. 38, no. 2, pp. 528-533, 2008.

[3] L. Wu and W. X. Zheng, "Weighted $H_{\infty}$ model reduction for linear switched systems with time-varying delay," Automatica, vol. 45, no. 1, pp. 186-193, 2009.

[4] L. Zhang, E.-K. Boukas, and P. Shi, "Exponential $H_{\infty}$ filtering for uncertain discrete-time switched linear systems with average dwell time: a $\mu$-dependent approach," International Journal of Robust and Nonlinear Control, vol. 18, no. 11, pp. 1188-1207, 2008.

[5] X. Zhao, L. Zhang, and P. Shi, "Stability of a class of switched positive linear time-delay systems," International Journal of Robust and Nonlinear Control, vol. 23, no. 5, pp. 578-589, 2013.

[6] L. Zhang and H. Gao, "Asynchronously switched control of switched linear systems with average dwell time," Automatica, vol. 46, no. 5, pp. 953-958, 2010.

[7] L. Farina and S. Rinaldi, Positive Linear Systems: Theory and Applications, vol. 50, John Wiley \& Sons, 2011. 
[8] X. Chen, J. Lam, P. Li, and Z. Shu, "L1-induced norm and controller synthesis of positive systems," Automatica, vol. 49, no. 5, pp. 1377-1385, 2013.

[9] J.-E. Feng, J. Lam, P. Li, and Z. Shu, "Decay rate constrained stabilization of positive systems using static output feedback," International Journal of Robust and Nonlinear Control, vol. 21, no. 1, pp. 44-54, 2011.

[10] P. Li, J. Lam, and Z. Shu, " $H_{\infty}$ positive filtering for positive linear discrete-time systems: an augmentation approach," IEEE Transactions on Automatic Control, vol. 55, no. 10, pp. 23372342, 2010

[11] P. Li, J. Lam, Z. Wang, and P. Date, "Positivity-preserving $H_{\infty}$ model reduction for positive systems," Automatica, vol. 47, no. 7, pp. 1504-1511, 2011.

[12] O. Mason and R. Shorten, "On linear copositive Lyapunov functions and the stability of switched positive linear systems," IEEE Transactions on Automatic Control, vol. 52, no. 7, pp. 13461349, 2007.

[13] M. A. Rami and F. Tadeo, "Controller synthesis for positive linear systems with bounded controls," IEEE Transactions on Circuits and Systems II: Express Briefs, vol. 54, no. 2, pp. 151-155, 2007.

[14] X. Zhao, P. Shi, and L. Zhang, "Asynchronously switched control of a class of slowly switched linear systems," Systems \& Control Letters, vol. 61, no. 12, pp. 1151-1156, 2012.

[15] X. Liu and C. Dang, "Stability analysis of positive switched linear systems with delays," IEEE Transactions on Automatic Control, vol. 56, no. 7, pp. 1684-1690, 2011.

[16] S. Fadali and S. Jafarzadeh, "Stability analysis of positive interval type-2 TSK systems with application to energy markets," IEEE Transactions on Fuzzy Systems, vol. 22, no. 4, pp. 1031-1038, 2013.

[17] L. Imsland, G. O. Eikrem, and B. A. Foss, "A state feedback controller for a class of nonlinear positive systems applied to stabilization of gas-lifted oil wells," Control Engineering Practice N, vol. 3, pp. 7-15, 2006.

[18] S. K. Nguang and P. Shi, "Fuzzy $H_{\infty}$ output feedback control of nonlinear systems under sampled measurements," Automatica, vol. 39, no. 12, pp. 2169-2174, 2003.

[19] O. Mason and M. Verwoerd, "Observations on the stability properties of cooperative systems," Systems \& Control Letters, vol. 58, no. 6, pp. 461-467, 2009.

[20] H. R. Feyzmahdavian, T. Charalambous, and M. Johansson, "Exponential stability of homogeneous positive systems of degree one with time-varying delays," IEEE Transactions on Automatic Control, vol. 59, no. 6, pp. 1594-1599, 2014.

[21] X. Ding, L. Shu, and X. Liu, "On linear copositive Lyapunov functions for switched positive systems," Journal of the Franklin Institute, vol. 348, no. 8, pp. 2099-2107, 2011.

[22] A. Jadbabaie, J. Lin, and A. S. Morse, "Coordination of groups of mobile autonomous agents using nearest neighbor rules," IEEE Transactions on Automatic Control, vol. 48, no. 6, pp. 988-1001, 2003.

[23] X. Zhao, L. Zhang, P. Shi, and M. Liu, "Stability of switched positive linear systems with average dwell time switching," Automatica, vol. 48, no. 6, pp. 1132-1137, 2012.

[24] X. Zhao, X. Liu, S. Yin, and H. Li, "Improved results on stability of continuous-time switched positive linear systems," Automatica, vol. 50, no. 2, pp. 614-621, 2014.

[25] A. S. Morse, "Supervisory control of families of linear setpoint controllers - part 1: exact matching," IEEE Transactions on Automatic Control, vol. 41, no. 10, pp. 1413-1431, 1996.
[26] D. Liberzon, Switching in Systems and Control, Springer, 2003.

[27] H. Liu, Y. Shen, and X. Zhao, "Asynchronous finite-time $\mathrm{H}_{\infty}$ control for switched linear systems via mode-dependent dynamic state-feedback," Nonlinear Analysis: Hybrid Systems, vol. 8, no. 1, pp. 109-120, 2013.

[28] J. Zhang, Z. Han, H. Wu, and J. Huang, "Robust stabilization of discrete-time positive switched systems with uncertainties and average dwell time switching," Circuits, Systems, and Signal Processing, vol. 33, no. 1, pp. 71-95, 2014.

[29] X. Zhao, L. Zhang, P. Shi, and M. Liu, "Stability and stabilization of switched linear systems with mode-dependent average dwell time," IEEE Transactions on Automatic Control, vol. 57, no. 7, pp. 1809-1815, 2012.

[30] J. Lian and J. Liu, "New results on stability of switched positive systems: an average dwell-time approach," IET Control Theory and Applications, vol. 7, no. 12, pp. 1651-1658, 2013.

[31] X. Zhao, S. Yin, H. Li, and B. Niu, "Switching stabilization for a class of slowly switched systems," IEEE Transactions on Automatic Control, 2014. 


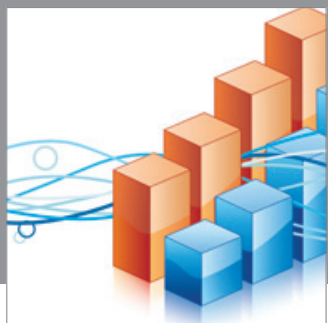

Advances in

Operations Research

mansans

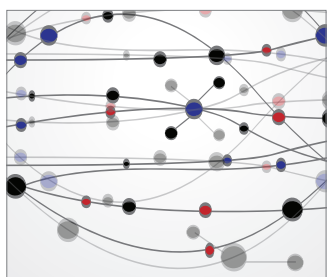

The Scientific World Journal
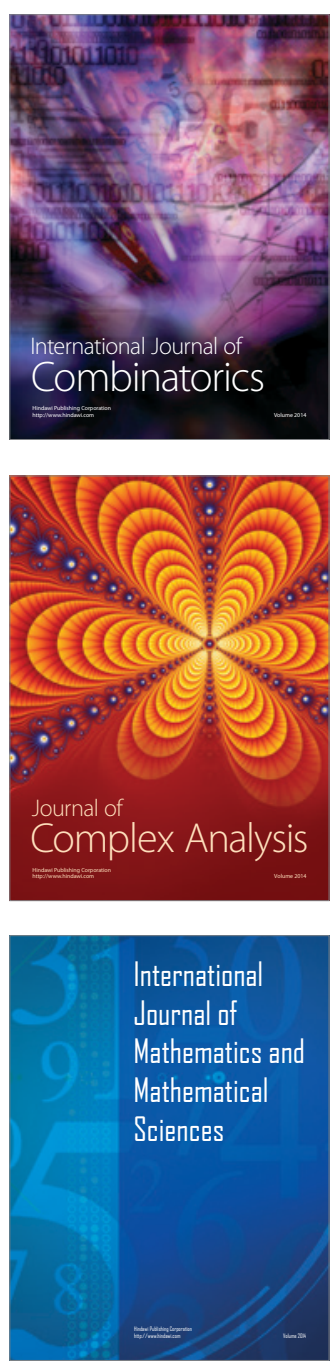
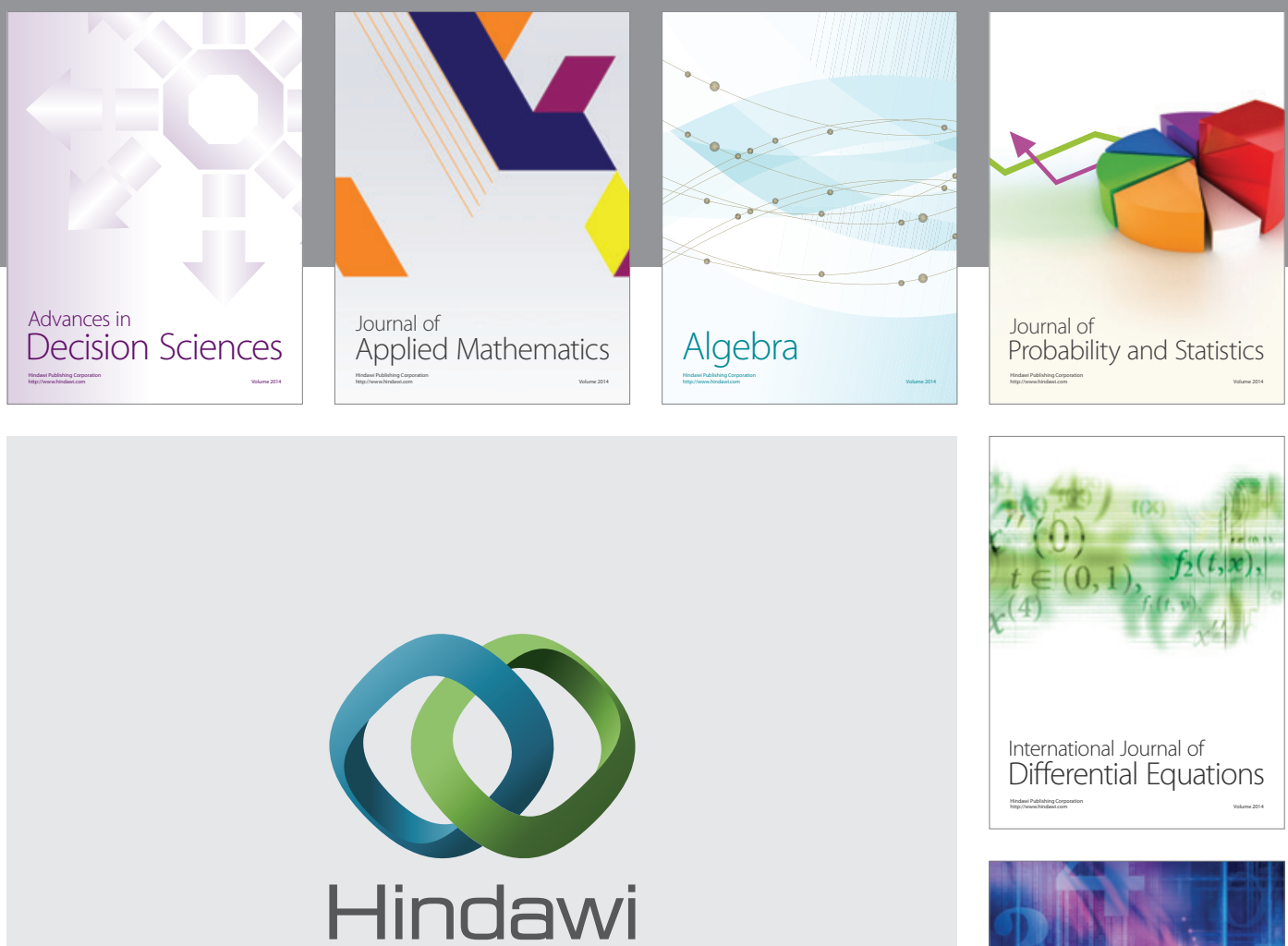

Submit your manuscripts at http://www.hindawi.com
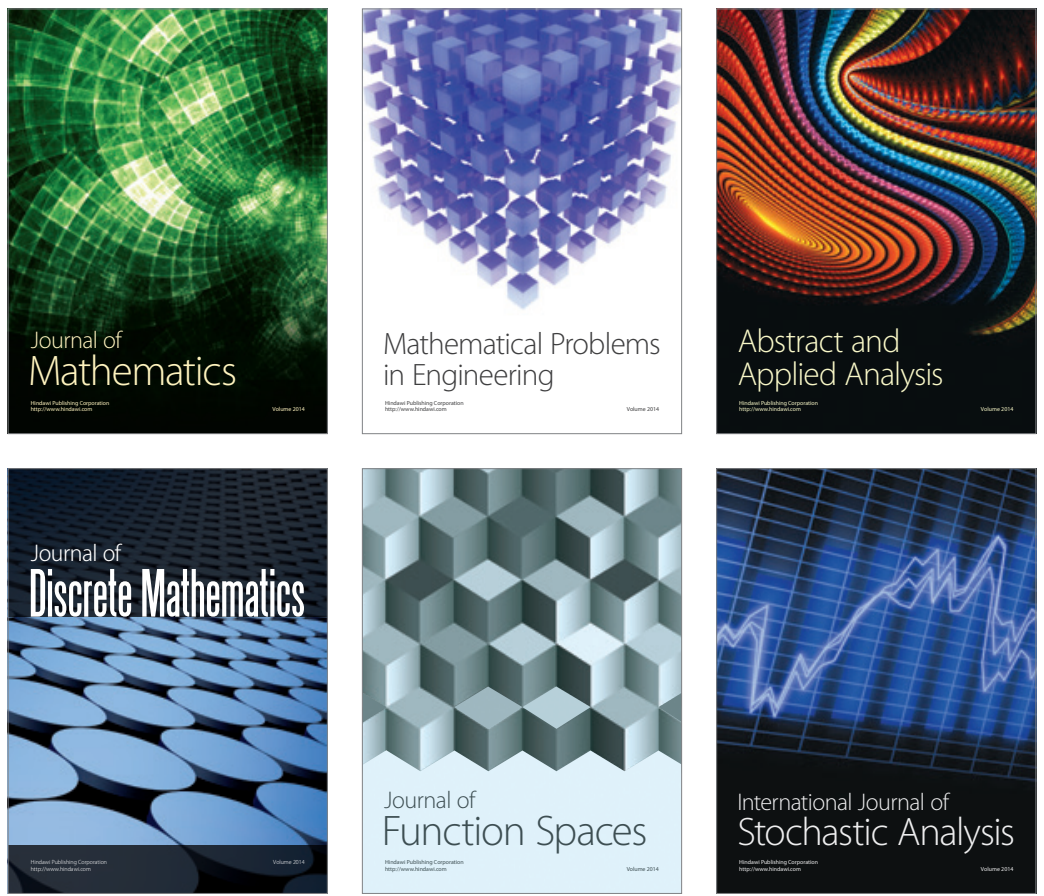

Journal of

Function Spaces

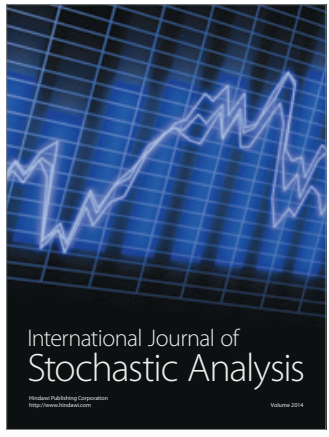

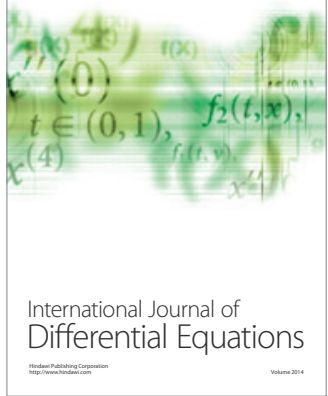
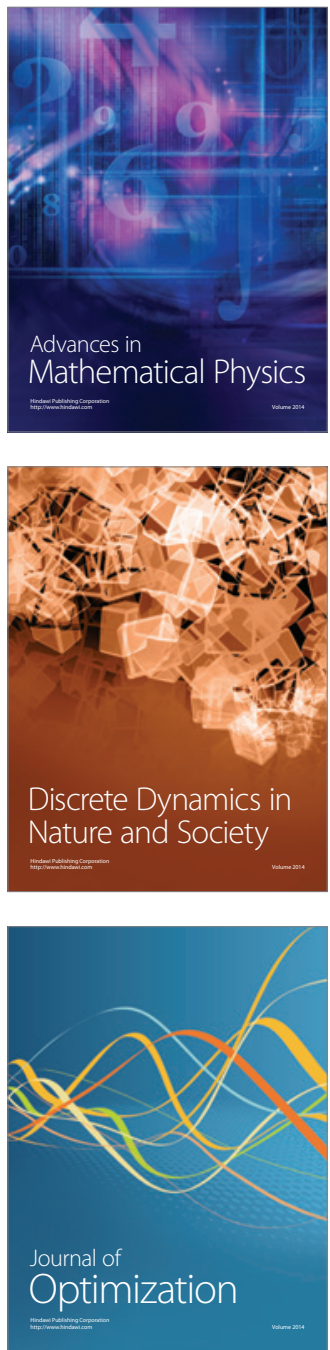